\title{
An Early Presentation of Neurosyphilis Manifesting as Cranial Polyneuropathies: A Case Report
}

\author{
Abinayaa P. Ravichandran ${ }^{\mathrm{a}}$, Katie Reming ${ }^{\mathrm{b}}$, Keithan Sivakumar ${ }^{\mathrm{c}}$, Hussam A. Yacoub ${ }^{\mathrm{a}, \mathrm{b}, \mathrm{d}}$
}

\begin{abstract}
Neurosyphilis (NS) is a rarely reported disease, with neurological manifestations occurring in the late stages of the infection. The incidence of NS has steadily increased, particularly with increased cases of human immunodeficiency virus (HIV) infection among homosexual partners, and can occur at any stage of the disease. In this case report, we present a 53-year-old homosexual man with HIV who presented with a gradual onset of vertigo over a course of 2 weeks, followed by gradual onset of multiple cranial neuropathies. Magnetic resonance imaging of the brain revealed pathological enhancement of multiple cranial nerves. Laboratory workup revealed reactive Venereal Disease Research Laboratory dilutional titer in the cerebrospinal fluid, supporting the diagnoses of NS. He was treated with intravenous penicillin with gradual resolution of symptoms. Our case illustrates that NS can occur with no known prior evidence or manifestation of the infection. The diagnosis of NS should be entertained in individuals with a known history of HIV and unexplained multiple cranial neuropathies.
\end{abstract}

Keywords: Syphilis; Neurosyphilis; Cranial neuropathies

\section{Introduction}

In the modern era, neurosyphilis (NS) is often a challenging diagnosis due to its rarity and diverse presentation. There has been a steady reemergence in the incidence of NS over the past two decades, particularly with the increase in prevalence of human immunodeficiency virus (HIV) infection among ho-

Manuscript submitted December 12, 2020, accepted February 15, 2021

Published online April 24, 2021

aLehigh Valley Hospital and Health Network, 1250 S Cedar Crest Blvd, Suite 405, Allentown, PA 18103, USA

${ }^{b}$ Morsani College of Medicine, University of South Florida, 12901 Bruce B Downs Blvd, Tampa, FL 33612, USA

'Department of Neurology, University of Toronto, 27 King's College Circle, Toronto, Ont. M5S 1A1, Canada

${ }^{\mathrm{d} C}$ Corresponding Author: Hussam A. Yacoub, Lehigh Valley Hospital and Health Network, 1250 S Cedar Crest Blvd, Suite 405, Allentown, PA 18103, USA. Email: Hussam_A.Yacoub@lvhn.org

doi: https://doi.org/10.14740/jnr650 mosexual partners. This case report describes an uncommon presentation of NS in an immunocompetent HIV-positive homosexual man who presented with multiple cranial neuropathies and headache.

\section{Case Report}

A 53-year-old homosexual man presented with a gradual onset of vertigo over a course of 2 weeks along with unilateral right-sided ear pain, fullness and hearing loss. He also experienced right-sided intermittent, throbbing headache and postnasal drip. The patient had a known history of HIV treated with antiretroviral therapy (ARVT), elvitegravir-cobicistatemtricitabine-tenofovir, with an undetectable viral load and a CD4 count of $847 / \mathrm{mm}^{3}$. Other comorbidities included hyperlipidemia, hypertension, pre-diabetes, factor- $V$ Leiden mutation and a history of deep vein thrombosis. The patient denied recent infections, fever, visual disturbance, neck pain/stiffness, or rash. Evaluation by otorhinolaryngology revealed minimal fluid in the middle ear compartment bilaterally on otoscopic examination. Audiometric evaluation revealed bilateral sensorineural hearing loss, with the right ear worse than the left by 10 decibels. Treatment was initiated with an oral prednisone taper, intranasal fluticasone and fexofenadine with minimal improvement of symptoms.

Three weeks later, the patient presented to the emergency department with right-sided facial paralysis and paresthesia. Neurological examination revealed right-sided wide palpebral fissure and inability to close the eyelid, flattening of the nasolabial angle, and decreased sensation to light touch in the distribution of the maxillary and mandibular trigeminal nerve branches. The remainder of the examination was unrevealing. Magnetic resonance imaging (MRI) of the brain revealed abnormal enhancement of the right facial nerve (Fig. 1) and subtle enhancement of the left facial nerve at the fundus of the left internal auditory canal (Fig. 2), with no cerebellopontine angle or intracranial masses, acute infarction, or hydrocephalus. Serology testing was negative for Lyme antibodies. Syphilis screening was not performed at this time. A presumed diagnosis of Bell's palsy was made, and the facial paralysis significantly improved. The patient continued to experience facial hypoesthesia, hearing loss, vertigo and intermittent headaches.

Over the following 4 weeks, the patient continued to have worsening headaches associated with occasional vomiting. He was treated with a tapering course of prednisone along with 


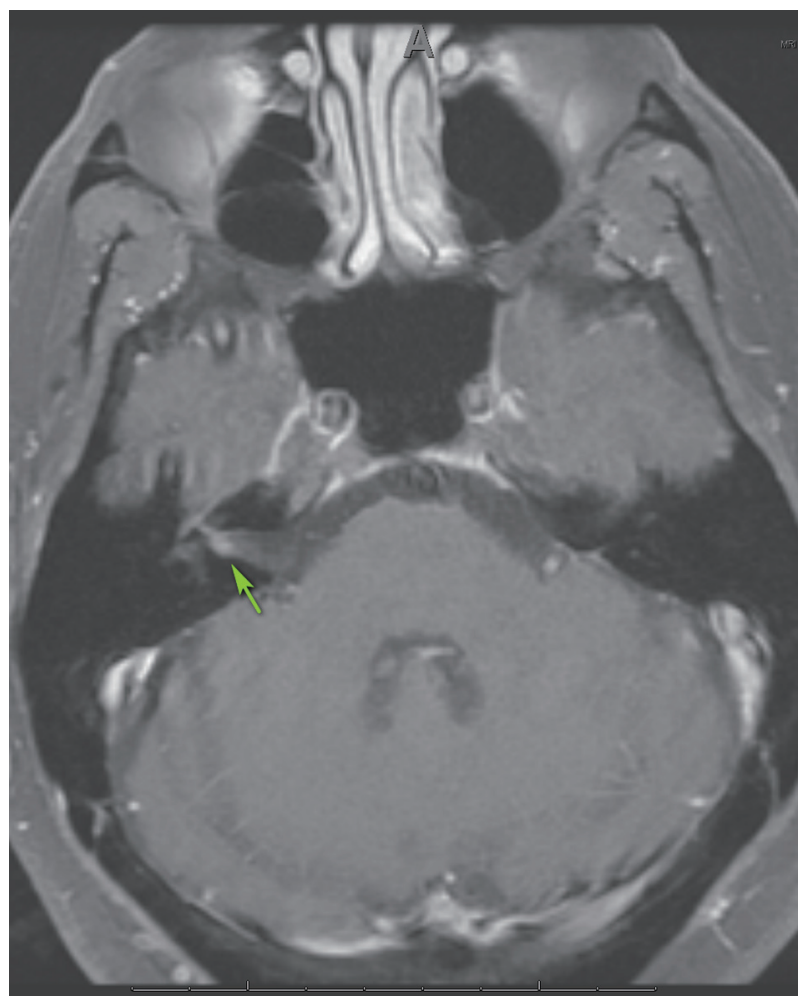

Figure 1. Axial MRI of the brain, T1 with gadolinium showing enhancement of $\mathrm{CN}$ VII on the right (arrow). MRI: magnetic resonance imaging; $\mathrm{CN}$ : cranial nerve.

oral acyclovir. Two days later, he experienced painless loss of peripheral vision. On ophthalmological evaluation, visual acuity was $20 / 50$ on the right, with an inferior temporal quadrant visual field deficit, loss of color vision and an afferent pupillary defect. On fundoscopic examination, the patient was found to have severe edema of the right optic nerve with evidence of hemorrhage at the margin. The remaining neurological examination was unremarkable.

Blood work revealed a white blood cell count (WBC) of $9.1 \times 10^{9} / \mathrm{L}$ and CD4 T cell count of $847 / \mathrm{mm}^{3}$, with an undetectable HIV viral load. Autoimmune testing was positive for antinuclear antibodies, with a titer of 1:80, and a rheumatoid factor titer of 1:211. Extensive immunological workup including anti-cytoplasmic antibody, double stranded DNA antibody, anti-Ro, anti-La, Smith auto-antibody, SCL 70 antibody and myeloperoxidase antibody was unrevealing. Serological testing was reactive for rapid plasma reagin (RPR), with titers of 1:256. West Nile virus, viral hepatitis and a repeat Lyme assay were negative. Cerebrospinal fluid (CSF) analysis revealed a clear, colorless fluid with an opening pressure of $17 \mathrm{~cm} \mathrm{H}_{2} \mathrm{O}$. Protein was elevated at $84 \mathrm{mg} / \mathrm{dL}$. CSF red blood cell count was 0 , and WBC count was elevated at $104 / \mathrm{mm}^{3}$ with a lymphocytic predominance of $96 \%$. Venereal Disease Research Laboratory (VDRL)-CSF dilutional titer was reactive at 1:4, suggestive of NS. Complete meningoencephalitis panel was unremarkable. MRI of the brain revealed expanded abnormal enhancements involving multiple cranial nerves, including the trigeminal nerve bilaterally (Fig. 3), the fundal and labyrin-

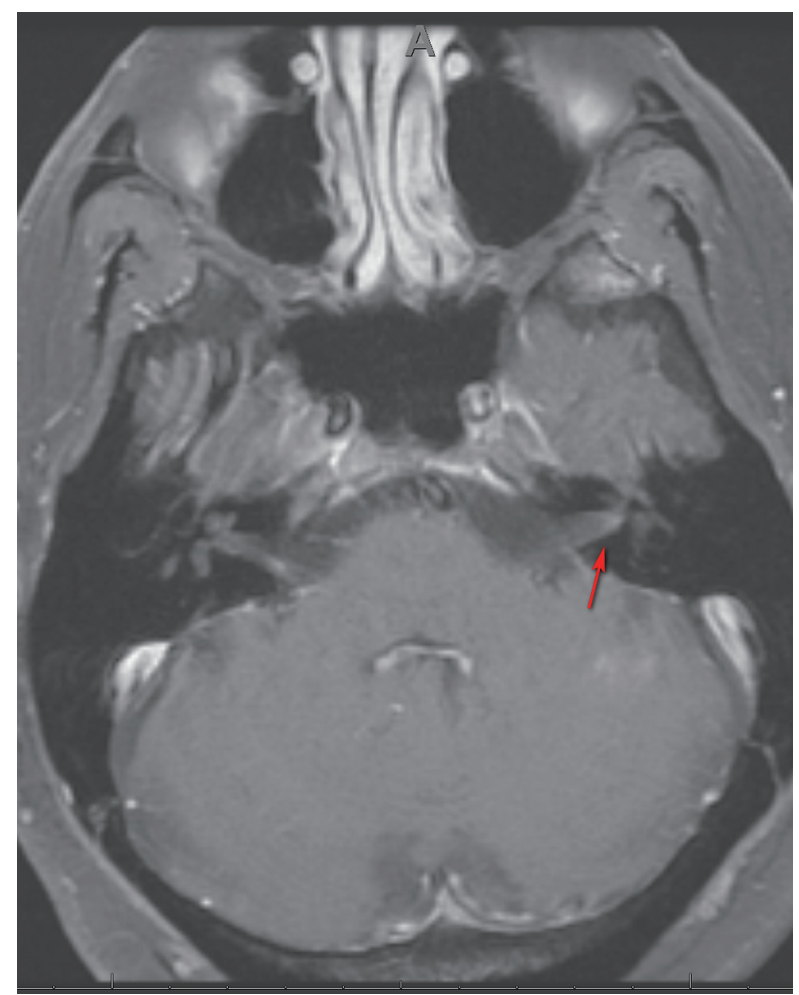

Figure 2. Axial MRI of the brain, $\mathrm{T} 1$ with gadolinium showing subtle enhancement of CN VII on the left (arrow). MRI: magnetic resonance imaging; $\mathrm{CN}$ : cranial nerve.

thine portions of the right facial nerve, and geniculate ganglion. Subtle enhancement was also seen at the fundus of the left internal auditory canal and right side of the optic chiasm (Fig. 4).

Treatment was initiated with IV penicillin G, 4 million units every $4 \mathrm{~h}$ for 14 days. Within $24 \mathrm{~h}$, the patient developed nausea, sinus tachycardia and a low grade fever. A JarischHerxheimer reaction was suspected and symptoms resolved with the use of acetaminophen and ondansetron. The patient was discharged on hospital day 5 with home infusion for the remainder of the antibiotic treatment. The facial paralysis completely resolved, with residual facial hypoesthesia, hearing loss and vision loss.

Over the course of several days, the patient reported subjective improvement of vision in the right eye by approximately $30 \%$. Ophthalmological evaluation revealed a visual acuity of 20/50 in the right eye with an afferent pupillary defect, absence of color vision and pale optic nerve on fundoscopic examination. Evaluation of the left eye was unrevealing. Ocular coherence tomography demonstrated damage to the peripheral retinal nerve fiber layer sparing the central vision. Hearing and facial weakness completely resolved with no residual deficits.

\section{Discussion}

Historically, NS has been described as a late manifestation of 


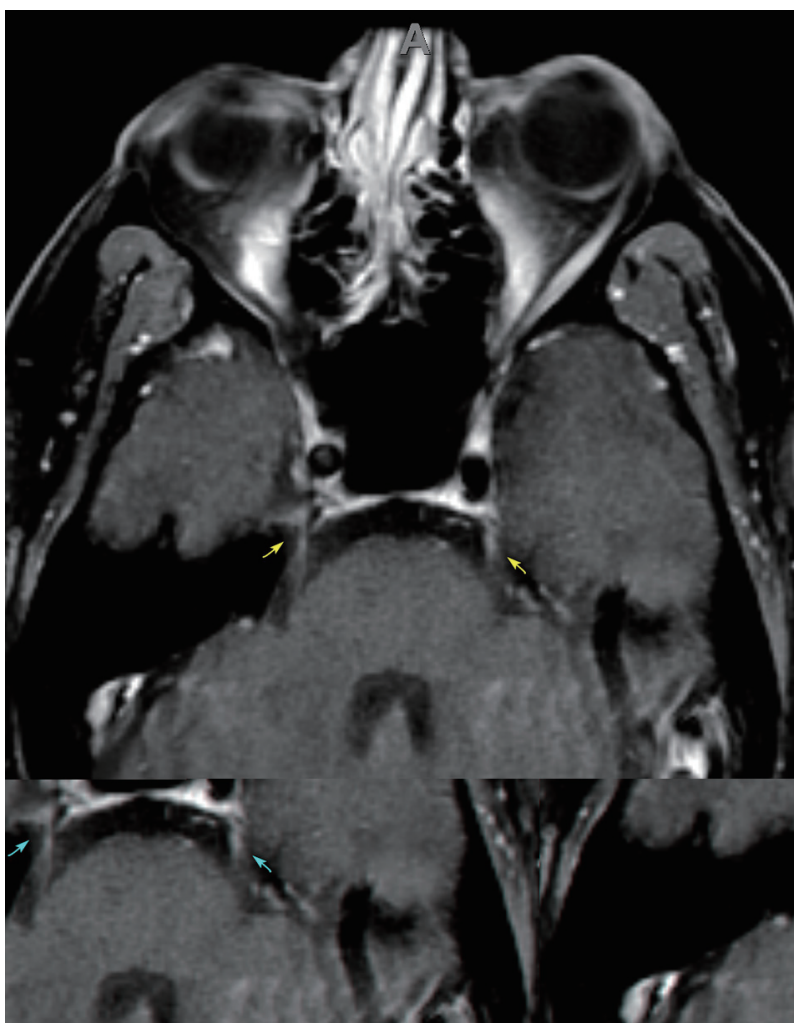

Figure 3. Axial MRI of the brain, T1 with gadolinium showing subtle enhancement of $\mathrm{CN} V$ bilaterally at the trigeminal cistern (arrows). MRI: magnetic resonance imaging; $\mathrm{CN}$ : cranial nerve.

the disease, occurring decades after the primary infection as tabes dorsalis and general paresis. NS is rarely reported in the United States with the availability and effectiveness of penicillin. The incidence of primary and secondary syphilis in the United States has increased substantially over the past two decades from 2.12 to 10.8 per 100,000 , with men who have sex with men (MSM) being the most at risk population [1,2]. Treponema pallidum ( $T$. pallidum), the spirochete bacterium that causes syphilis, can invade the central nervous system (CNS) at any stage of the infection $[3,4]$. The majority of individuals remain asymptomatic, with evidence of bacterium eradication without any pharmacotherapy [4]. Less than $2 \%$ of patients develop NS, with symptoms occurring months to decades after the initial infection [5].

Aseptic meningitis is one of the earliest manifestations of NS, with a prototypical constellation of symptoms including headache, photophobia and neck stiffness. This can progress to ocular syphilis and cranial neuropathies [3]. In a case series published by the Center for Disease Control, approximately $75 \%$ of patients had visual symptoms, ocular manifestation and headache, whereas only $6 \%$ and $12 \%$ had cranial neuropathies and acute meningitis, respectively [6]. Vascular complications such as stroke and hemorrhage have been reported in approximately $4 \%$ of patients $[6,7]$. Neurological manifestations of syphilis are more than twice as likely to occur in cases of HIV co-infection, with the prevalence of NS being $2.1 \%$ in HIV-positive versus $0.6 \%$ in HIV-negative patients [8]. There

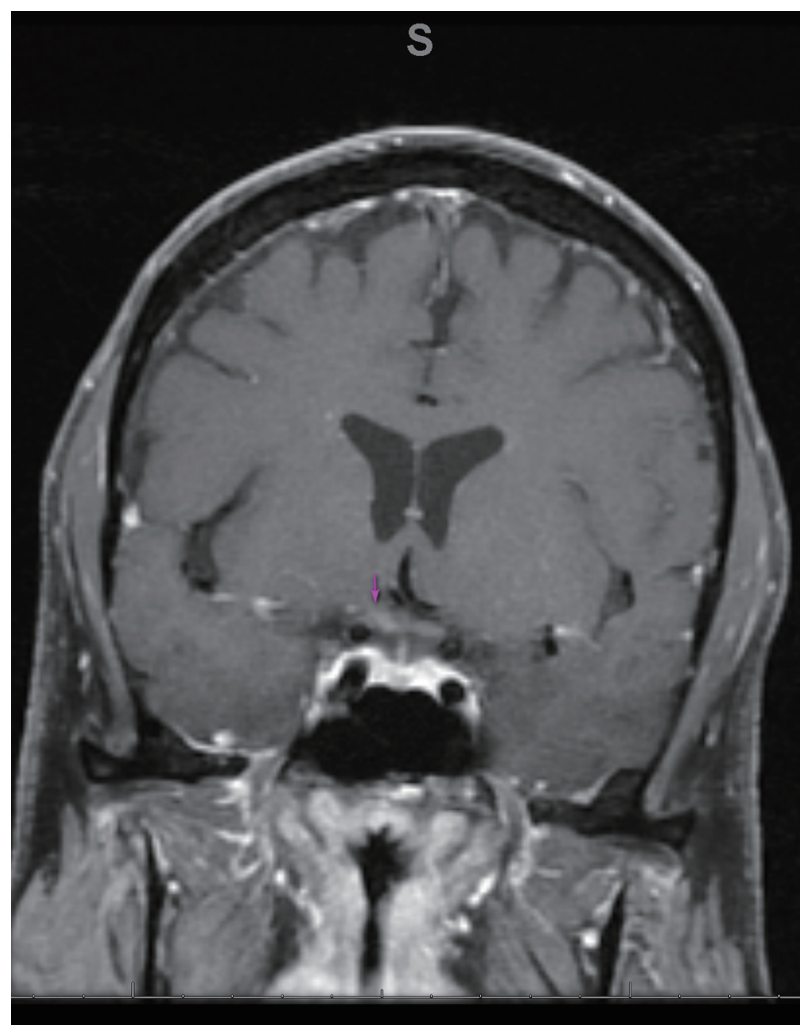

Figure 4. Coronal MRI of the brain, T1 with gadolinium showing enhancement on the right side of the optic chiasm (arrow). MRI: magnetic resonance imaging.

is also evidence of a positive correlation between the degree of immunosuppression and the likelihood of developing NS [3].

NS may present earlier and occur more frequently in patients with HIV [8, 9], and often has a fulminant course with overlapping disease stages compared to patients without an HIV co-infection [10]. Our patient presented with a headache and multiple cranial neuropathies, in the absence of fever or other signs of meningeal irritation. He did not have any symptoms suggestive of primary or secondary syphilis, supporting the notion that CNS infection with T. pallidum can occur at any stage. A prospective cohort study reported that syphilis could adversely affect the HIV viral load and CD4 count even in patients receiving ARVT, therefore increasing the risk of transmission [11].

A study in Los Angeles reported a $2.1 \%$ increase in the incidence of NS among individuals infected with HIV compared to $0.6 \%$ in the non-HIV cohort between 2001 and 2004 . The authors speculated a greater risk of developing NS with serum RPR titers $>1: 32$ and/or CD4 count $<350 / \mathrm{mm}^{3}$ [8]. Another study by Ghanem et al in 2008 reported that a CD4 $<350 / \mathrm{mm}^{3}$ is a strong predictor of developing both symptomatic and asymptomatic NS. The CD4 count in this cohort ranged between 72 and $345 / \mathrm{mm}^{3}$ in individuals with NS [12]. Our patient's HIV infection was well controlled with ARVT, indicated by an undetectable viral load and a CD4 T-cell count of $847 / \mathrm{mm}^{3}$. Although Ghanem et al reported a synergistic relationship with increased risk of NS in HIV patients with a 
low CD4 count [12], our patient developed NS despite a nonimmunocompromised state.

The increased susceptibility to NS in individuals with HIV may not be predominantly proportional to the degree of immunosuppression [3]. This suggests that being immunosuppressed rather than immunocompromised may have played a role in our patient's increased susceptibility to acquiring NS. Successful treatment with anti-retroviral therapy has been posited to effectively downregulate both the innate and acquired immune system's ability to respond to T. pallidum [13]. This mechanism may help explain the relatively rapid progression to NS in a previously asymptomatic patient with a well-controlled HIV infection.

Diagnostic workup for NS includes serological testing, CSF analysis and concomitant neuroimaging. Patients with reactive non-treponemal tests should undergo confirmatory treponemal assays as false positives to the former have been reported in many HIV patients. CSF VDRL is highly specific but not sensitive. A positive CSF VDRL in the setting of clinical signs and symptoms is diagnostic of NS. If VDRL is negative, CSF fluorescent treponemal antibody absorbed (FTA-ABS) testing coupled with serology and clinical signs are required to establish the diagnosis [14]. MRI of the brain often reveals enhancing or non-enhancing cortical lesions, a pattern suggestive of encephalitis, with or without adjacent meningeal enhancement and cerebral infarctions [15]. The varied clinical presentation in our patient prompted repeat imaging, which revealed interval progression and multiple cranial nerve enhancements. Magnetic resonance angiography and spinal cord imaging are warranted if there are signs of vasculitis and posterior column involvement, respectively. Alternative diagnoses of conditions with overlapping imaging features including viral or other bacterial infections, demyelination, or inflammatory diseases should be entertained.

Parenteral penicillin $\mathrm{G}$ is the treatment of choice for NS. The dosage, duration and formulation vary among the different stages and systemic manifestations. Jarisch-Herxheimer reaction, an allergic febrile response to treatment, occurs most frequently in the early stages of NS, presumably due to extensive bacterial burden. NS patients with underlying HIV are treated similarly to those without the co-infection. Follow-up with repeat serologies at 6 and 12 months is recommended in individuals with clinical resolution of symptoms [14].

\section{Learning points}

NS can present with atypical symptoms including multiple cranial neuropathies as an early manifestation of the disease, as the spirochete can invade the CNS at any stage of the infection. Our unique case describes an HIV patient with adequate CD4 count who developed neurological manifestations with no evidence of primary or secondary syphilis. The diagnosis of NS should be entertained in individuals with unexplained multiple cranial neuropathies, particularly in HIV-infected patients, even when the CD4 count is $>350 / \mathrm{mm}^{3}$. The uncertainties surrounding the pathogenesis of co-infection with NS and HIV need to be further explored.

\section{Acknowledgments}

None to declare.

\section{Financial Disclosure}

None to declare.

\section{Conflict of Interest}

None to declare.

\section{Informed Consent}

Informed consent was obtained from the patient verbally and there are no identifying features in the paper.

\section{Author Contributions}

All authors contributed equally to the production of this manuscript. The entire manuscript was reviewed and edited by all individuals listed.

\section{Data Availability}

The authors declare that data supporting the findings of this study are available within the article. All data were extrapolated from referenced studies and those studies may be reviewed for specific details pertaining to their data collection.

\section{References}

1. Centers for Disease Control and Prevention. Syphilis surveillance supplemental slides, 2014-2018: Technical Notes. Atlanta: U.S. Department of Health and Human Services; 2020.

2. Schmidt R, Carson PJ, Jansen RJ. Resurgence of Syphilis in the United States: an assessment of contributing factors. Infect Dis (Auckl). 2019;12:1178633719883282.

3. Hobbs E, Vera JH, Marks M, Barritt AW, Ridha BH, Lawrence D. Neurosyphilis in patients with HIV. Pract Neurol. 2018;18(3):211-218.

4. Peeling RW, Mabey D, Kamb ML, Chen XS, Radolf JD, Benzaken AS. Syphilis. Nat Rev Dis Primers. 2017;3:17073.

5. de Voux A, Kidd S, Torrone EA. Reported Cases of Neurosyphilis Among Early Syphilis Cases-United States, 2009 to 2015. Sex Transm Dis. 2018;45(1):39-41.

6. Symptomatic early neurosyphilis among HIV-positive men who have sex with men, four cities, United States, January 2002-June 2004. Centers for Disease Control and 
Prevention. Atlanta, GA.

7. Koh M, Kashiwazaki D, Yamatani K, Kuroda S. [Intracranial hemorrhage caused by neurosyphilis: a case report]. No Shinkei Geka. 2014;42(8):711-716.

8. Taylor MM, Aynalem G, Olea LM, He P, Smith LV, Kerndt PR. A consequence of the syphilis epidemic among men who have sex with men (MSM): neurosyphilis in Los Angeles, 2001-2004. Sex Transm Dis. 2008;35(5):430-434.

9. Tuddenham S, Ghanem KG. Emerging trends and persistent challenges in the management of adult syphilis. BMC Infect Dis. 2015;15:351.

10. Kurtz SD, Rollin F. Ocular syphilis in a patient with HIV. JAAPA. 2014;27(4):32-35.

11. Jarzebowski W, Caumes E, Dupin N, Farhi D, Lascaux AS, Piketty C, de Truchis P, et al. Effect of early syphilis infection on plasma viral load and CD4 cell count in human immunodeficiency virus-infected men: results from the FHDH-ANRS CO4 cohort. Arch Intern Med. 2012;172(16):1237-1243.

12. Ghanem KG, Moore RD, Rompalo AM, Erbelding EJ, Zenilman JM, Gebo KA. Neurosyphilis in a clinical cohort of HIV-1-infected patients. AIDS. 2008;22(10):11451151.

13. Rekart ML, Ndifon W, Brunham RC, Dushoff J, Park SW, Rawat S, Cameron CE. A double-edged sword: does highly active antiretroviral therapy contribute to syphilis incidence by impairing immunity to Treponema pallidum? Sex Transm Infect. 2017;93(5):374-378.

14. Centers for Disease Control and Prevention. Treatment guideline for Syphilis. 2015.

15. Brightbill TC, Ihmeidan IH, Post MJ, Berger JR, Katz DA. Neurosyphilis in HIV-positive and HIV-negative patients: neuroimaging findings. AJNR Am J Neuroradiol. 1995;16(4):703-711. 